Revue des patrimoines

19 | 2012

Patrimoines et conservation préventive. Pratiques comparées et nouveaux enjeux

\title{
Formation des archéologues à la conservation préventive : pour une démarche de conservation
}

\section{Silvia Païn}

\section{(2) OpenEdition}

Journals

Édition électronique

URL : http://journals.openedition.org/insitu/9820

DOI : $10.4000 /$ insitu. 9820

ISSN : 1630-7305

Éditeur

Ministère de la culture

\section{Référence électronique}

Silvia Paiin, «Formation des archéologues à la conservation préventive : pour une démarche de conservation », In Situ [En ligne], 19 | 2012, mis en ligne le 21 septembre 2012, consulté le 30 avri 2019. URL : http://journals.openedition.org/insitu/9820 ; DOI : 10.4000/insitu.9820

Ce document a été généré automatiquement le 30 avril 2019

\section{cc) (†)}

In Situ Revues des patrimoines est mis à disposition selon les termes de la licence Creative Commons Attribution - Pas d'Utilisation Commerciale - Pas de Modification 4.0 International. 


\title{
Formation des archéologues à la conservation préventive : pour une démarche de conservation
}

\author{
Silvia Païn
}

\section{Introduction}

1 La sensibilité aux problématiques de conservation du mobilier archéologique s'exprime, depuis quelques années, de façon affirmée. La professionnalisation de l'archéologie et les contraintes de l'archéologie préventive ont provoqué une augmentation des volumes de mobilier à traiter, mais aussi une prise en compte de la place de la conservation préventive dans le processus de traitement du mobilier et de l'importance de sa gestion par un personnel dédié. Dans ce contexte, il a paru nécessaire d'engager des actions de formation à la conservation préventive, qui se distinguent à plusieurs titres de celles qui sont destinées à des professionnels d'autres secteurs patrimoniaux. En effet, si les avancées méthodologiques dans la prévention suscitent aussi un intérêt croissant dans les domaines des musées, des archives, des monuments..., de tout temps la conservation a été leur mission principale, alors que pour l'archéologie, c'est, le plus souvent, la recherche scientifique qui a été mise au premier plan, la conservation après la fouille étant habituellement confiée à l'administration des monuments historiques, pour ce qui est des vestiges immeubles, et aux musées, pour ce qui est des objets. D'autre part, dans la plupart des autres secteurs, les biens culturels à préserver sont déjà reconnus en tant que tels et ont pris leur forme définitive, alors que le mobilier archéologique nécessite un travail d'identification et d'étude qui passe par des étapes où la manipulation du mobilier, effectuée par les archéologues eux-mêmes, est indispensable.

La sensibilisation et la formation à la conservation préventive en archéologie sont donc un enjeu essentiel : l'auteur de ces lignes, en 25 ans de vie professionnelle, dont la plupart au sein d'un service archéologique de collectivité, a animé une dizaine de sessions 
formalisées, répondu à des demandes ponctuelles de conseil ou d'assistance à de multiples reprises et les échanges avec les collègues archéologues sur les questions de conservation sont quotidiens. C'est le fruit des réflexions que ces expériences ont suscitées qui sera exposé ici. Une formation efficace ne se construit pas en faisant abstraction du public à former et de ses attentes : il convient d'examiner, tout d'abord, son niveau de sensibilisation dans le domaine de la conservation et ses connaissances préalables. Nous verrons ensuite les modalités concrètes des actions de formation qui ont $\mathrm{pu}$ avoir lieu, ainsi que leur contenu. Nous tenterons ensuite d'exposer quelques problématiques liées à la transmission de connaissances dans ce domaine et à l'évaluation de l'efficacité des actions, pour conclure sur la possibilité, au-delà des conseils factuels et des "recettes", de susciter lors de ces formations une véritable démarche de conservation cohérente, depuis le terrain jusqu'au stockage définitif.

\section{Connaissances et sensibilité préalables à la formation}

Former des archéologues à la conservation implique nécessairement de se demander quelles sont les connaissances préalables dans le domaine concerné, mais aussi de cerner l'importance donnée à celui-ci et les éventuelles résistances qui pourraient se faire jour. Dans le cas de l'archéologie, nous pouvons distinguer trois aspects qui nous semblent devoir être pris en compte : l'absence de sensibilisation au cours de la formation initiale, la hiérarchie relative entre dimension scientifique et dimension patrimoniale et les résistances s'exprimant dans des attitudes fatalistes vis-à-vis de la possibilité de la conservation du mobilier, soit intrinsèquement, soit du fait du manque de moyens.

\section{La formation initiale}

4 Ceux qui ont été confrontés aux problématiques de conservation en archéologie ont constaté avec regret que la formation initiale des archéologues ne comprend pas d'enseignements spécifiques sur la chimie des matériaux archéologiques, leur altération dans le temps et les mécanismes de dégradation auxquels ils sont soumis, aussi bien dans le milieu d'enfouissement que dans le milieu atmosphérique après leur exhumation.

5 L'archéologue qui arrive sur un terrain de fouilles, selon sa spécialité et ses centres d'intérêt, sera peut-être plus ou moins sensibilisé à un matériau particulier, mais on ne peut être assuré qu'il a ne serait-ce qu'une vue d'ensemble sur les matériaux les plus courants, ni même qu'il sache les reconnaître dans leur état dégradé. Si l'altération des objets est abordée, c'est uniquement pour traiter les éventuels problèmes que cette dégradation posera pour l'étude scientifique (patine naturelle ou artificielle, disparition de certains composants qui ne pourront plus être détectés par l'analyse,...), mais pas nécessairement pour faire prendre conscience de la fragilité des matériaux, du fait que certains objets ne sont pas directement lisibles après la fouille, ou des effets que les manipulations nécessaires à l'étude pourraient induire sur la conservation à long terme. Certains archéologues croient ainsi sincèrement que la découverte du mobilier entraîne de par ce fait même sa conservation. En particulier, les dégradations dues à un séjour trop prolongé sur le terrain, justifié par les nécessités de la fouille, de la photographie de la structure ou du relevé sont, le plus souvent, sous-estimées ou considérées comme un fait sur lequel on n'a pas de prise. 
6 Pour ce qui concerne le post-fouille, rien n'est dit non plus à l'étudiant en archéologie sur les problématiques de conservation du mobilier exhumé, sur les effets que ses propres pratiques d'étude peuvent avoir sur celle-ci, non plus que sur les traitements de conservation-restauration. Ces derniers sont peu abordés, que ce soit du point de vue de leur intérêt pour l'étude et l'identification des objets (nettoyage d'objets métalliques, par exemple), ou des précautions nécessaires vis-à-vis de certaines méthodes d'analyse ou d'examen, dans la mesure où quelques interventions, telle que la consolidation, peuvent fausser certains résultats ou obérer certaines possibilités d'étude.

7 De surcroît, la formation initiale est centrée, la plupart du temps, sur des contenus sur l'archéologie des civilisations anciennes et ne comprend pas beaucoup d'enseignements méthodologiques portant sur le caractère patrimonial des découvertes archéologiques, perçues essentiellement comme des objets de science.

\section{Recherche archéologique et conscience patrimonial}

Au cours de sa vie professionnelle, l'archéologue est pleinement conscient d'assumer une responsabilité scientifique vis-à-vis de l'enregistrement des vestiges détruits (structures, stratigraphie) ainsi que dans le choix de ramassage du mobilier; il n'est pas sûr que la dimension patrimoniale de son activité (impliquant des soucis de conservation) lui apparaisse toujours de façon aussi évidente. La prééminence donnée au terrain et aux « structures » dans le raisonnement archéologique, si elle conduit les professionnels à se soucier de la conservation des sites non encore fouillés, ne favorise pas l'extension de cette préoccupation à la prise en charge des problématiques de conservation du mobilier après la fouille et le rendu du rapport.

Bruno Dufaÿ, lui-même archéologue, a tenté, dans sa contribution aux Assises de la conservation archéologique de Bourges ${ }^{1}$, d'expliquer ce phénomène. Il constate, pour les regretter, une « indifférence totale vis-à-vis de la matérialité des objets étudiés » et un « extrême mépris pour les objets en question, envisagés uniquement comme porteurs d'informations et comme résidus de la recherche ", et y voit trois raisons principales : la dilution de la notion d'objet, la disparition de l'objet derrière l'information qu'il véhicule et l'essor du virtuel. Il propose une approche plus soucieuse de conservation, d'une part pour permettre l'émergence de pistes de recherche liées à la matérialité, d'autre part pour ne pas se couper d'une population pour qui les objets, au-delà de leur intérêt scientifique, sont source d'émotion.

Beaucoup d'archéologues ont pris conscience de l'intérêt de considérer la matérialité de l'objet et sa dimension patrimoniale, et ce sentiment est sans doute amené à se développer. Cependant, il n'est pas encore général : à la question que Nicole Rodrigues, archéologue municipale de la ville de Saint-Denis (Seine-Saint-Denis), posait, en 1990 : "L'archéologue est-il un " gérant du patrimoine » ou en est-il un des prédateurs? ", Bruno Dufaÿ répond en 1998 en disant que «l'attitude commune de l'archéologue est davantage celle d'un prédateur que d'un gestionnaire : après qu'il a fouillé (ou une fois son étude finie), il reste quelques résidus dont il ne se préoccupe guère $»^{3}$ et Bruno Desachy, conservateur du patrimoine, en 2008, que «les archéologues de terrain se soucient fréquemment très peu de conservation $»^{4}$.

11 Que l'on nous comprenne bien : il n'est pas dans notre intention de porter de jugement de valeur sur les archéologues ni de les accuser de quoi que ce soit, encore moins d'opposer archéologues et conservateurs-restaurateurs. On peut d'ailleurs dire que ceux-ci, 
réciproquement, à moins d'avoir aussi une formation ou une expérience en archéologie, se vouent à la conservation patrimoniale sans forcément toujours cerner la dimension scientifique... L'enjeu et l'intérêt de la conservation en archéologie n'est pas de choisir entre science et conservation, mais de concilier les deux objectifs en les optimisant. Pour cela, il faut d'abord comprendre les attentes et motivations de chacun.

\section{Résistances : fatalisme et manque de moyens} préventives ou curatives, ne sont pas apportées aux archéologues dans leur formation initiale, seuls un acte volontariste ou les hasards des rencontres peuvent les leur apporter au cours de leur vie professionnelle. Ceux qui n'y ont pas eu accès, pour ce qui concerne la conservation à long terme de matériaux fragiles, sont parfois gagnés par un certain fatalisme : par méconnaissance des " gestes qui sauvent ", ils considèrent la détérioration comme inéluctable.

D'autre part, une certaine culture de la pauvreté et du système $\mathrm{D}$, qui pouvait se justifier aux temps de l'archéologie bénévole sans budgets de fonctionnement, perdure parfois dans les esprits, même si les moyens réels de l'archéologie professionnelle ont considérablement augmenté depuis cette époque. L'idée que la conservation peut coûter cher et, surtout, qu'elle entrave nécessairement la fouille et fait perdre du temps est encore assez présente, alors même que, par ailleurs, les opérateurs d'archéologie préventive se dotent sans états d'âme de spécialistes coûteux dans d'autres domaines (géomorphologue, anthropologue, analyses ou datations...), et que bien d'autres activités sont au moins aussi chronophages («nettoyage photo », relevés pierre à pierre),... mais perçues comme plus légitimes.

14 Certains associent les deux facteurs: ainsi, pour justifier une analyse destructive, une archéologue, spécialiste des fers de trait médiévaux ${ }^{5}$, affirme que "ces objets, suivant leurs conditions de conservation, risquent de disparaître, à terme, sous l'effet de la corrosion, à moins de subir une opération de stabilisation coûteuse, pas toujours envisagée pour ce type d'artefacts.» Ici, l'idée que des mesures de conservation préventive, telles que la conservation dans un milieu exempt d'humidité, peuvent retarder l'échéance fatale, ne semble pas apparaître comme une alternative. Le caractère « coûteux » d'une stabilisation serait également à relativiser, s'agissant d'un traitement pouvant être mis en œuvre pour des lots d'objets simultanément (à titre de comparaison, le coût d'une datation C14 est comparable à celui du traitement de stabilisation d'une trentaine d'objets ferreux).

Tant qu'un certain nombre d'archéologues pensera que la dégradation post-fouille du mobilier est inéluctable, ou bien que les moyens de l'éviter sont hors de leur portée, il peut sembler difficile de mettre en place une démarche de conservation préventive, même des plus modestes... aux formateurs de jouer, donc, pour modifier cette image ! global qui résulte de cet exposé : il correspond toutefois à une situation réelle, dénoncée, comme on l'a vu plus haut, par des archéologues, dans des termes bien plus vifs que ceux que nous aurions employés... S'il nous a semblé utile de mettre en lumière ces différents aspects, c'est parce que ce sont eux qui déterminent la façon dont la formation sera reçue et sur quels ressorts nous pouvons jouer pour faire avancer la sensibilisation à la conservation en archéologie. 


\section{La formation à la conservation préventive en archéologie : émergence et modalités}

\section{Prise de conscience des besoins de formation}

17 L'émergence d'un souci de conservation du mobilier sur le long terme, en particulier chez des archéologues spécialistes des études de mobilier, se fait jour le plus souvent à l'occasion de la découverte d'un type de mobilier fragile, rare ou non lisible immédiatement. Le recours obligé à des spécialistes de la conservation-restauration pour leur traitement peut faciliter une prise de conscience des problématiques de conservation et un questionnement sur les façons de prélever, conditionner, manipuler,... pour pouvoir étudier plus tard. Nicole Rodrigues a souvent évoqué le rôle joué par les échanges et la collaboration avec les enseignants de la maitrise de sciences et techniques en Conservation-restauration des biens culturels de l'université de Paris 1 , dans l'expérience pionnière de l'Unité municipale d'archéologie de Saint-Denis ${ }^{6}$.

18 Un certain nombre de professionnels dans la communauté archéologique, les responsables des institutions où les archéologues exercent leur activité, ainsi que les pouvoirs publics ont, depuis quelques années, souvent pris conscience de l'intérêt que présente une sensibilisation à la conservation du mobilier ainsi que sa préservation à long terme, et les documents commandés par le ministère de la Culture sur les Centres de conservation et d'étude, pour ne prendre que cet exemple, témoignent de cette ambition ${ }^{7}$. Dans le même esprit, le ministère de la Culture, des services de collectivité territoriale et certains opérateurs ont proposé à leurs agents des formations sur la conservation préventive, sans forcément attendre une demande venue du terrain.

19 Enfin, le développement des postes de régisseurs des collections (qu'on appelle aussi « responsables du dépôt archéologique » ou "gestionnaires de mobilier») a facilité la prise en compte des problèmes de conservation dans les équipes. Le régisseur étant, souvent, archéologue de formation, il constitue l'interface entre les professionnels de la conservation matérielle et les chercheurs, pouvant faire passer dans la durée les pratiques apprises lors de formations nécessairement courtes et dont tous les archéologues ne peuvent bénéficier.

20 Parallèlement, les conservateurs-restaurateurs en archéologie, très concernés par le problème, ont depuis longtemps une démarche volontariste et que l'on pourrait qualifier parfois de prosélyte, voire de missionnaire, pour répandre la «bonne parole» et les "gestes qui sauvent » dans la communauté archéologique. Un premier exemple est, en 1993, une initiative conjointe de l'Association des conservateurs-restaurateurs en archéologie et du responsable de la formation continue à l'AFAN (Association pour les fouilles archéologiques nationales) pour rédiger un cahier de charges pour une formation en conservation préventive, qui a servi de cadre à des actions de formation organisées par différents laboratoires (UTICA et Archéologies, en particulier), ${ }^{8}$, un autre la rédaction de documents destinés aux archéologues de l'INRAP', mais aussi les initiatives récentes du CNFPT (www.cnfpt.fr).

21 Dans les équipes qui comptent un conservateur-restaurateur dans leurs rangs, telles que certains services de collectivité territoriale (ou l'INRAP), les formations se font souvent en 
interne, et les recommandations données ne sont pas de simples conseils, mais ont vocation à devenir des véritables normes de fonctionnement ${ }^{10}$.

Il y a donc une offre d'information et de formation en conservation préventive. Dans la mesure où la demande est rarement formulée de façon très précise, du fait même de la méconnaissance du domaine par ses futurs bénéficiaires, ces formations sont le plus souvent conçues et mises en œuvre par des conservateurs-restaurateurs en archéologie, et sont marquées par les modalités d'apprentissage qu'ils ont eux-mêmes expérimentées et par la bibliographie de leur domaine. Très souvent, à l'issue de ces formations, des documents sont distribués, qui reprennent les principaux conseils en matière de prélèvement sur le terrain, de traitement post-fouille (lavage, séchage) ou de conservation à long terme. Ceux-ci sont parfois réutilisés dans les documents normatifs des équipes de fouille ou des structures de conservation ${ }^{11}$.

\section{Contenus de la formation}

Le premier sujet traité dans la plupart des formations dispensées concerne la connaissance des matériaux altérés. En effet, on ne peut raisonnablement s'attendre à voir émerger un souci de conservation sur un mobilier dont la nature et la fragilité n'ont pas été identifiées : aussi, un premier niveau d'information en conservation préventive passe généralement par une observation commentée d'un échantillonnage des différents matériaux courants en archéologie, dans divers états de dégradation.

Il s'agit ensuite d'expliquer les facteurs d'altération: ceux responsables des altérations qui ont lieu pendant l'enfouissement, bien sûr, mais aussi ceux qui vont jouer lors de l'exhumation, puis sur le long terme, dans la phase d'étude et de stockage. De cet exposé découlent des préconisations pour l'action, la plupart du temps déclinées par famille de matériaux (organiques/inorganiques ; « sensibles (métaux, organiques)/peu sensibles »,... ).

Dans les formations proposées, on donnera le plus souvent des conseils pratiques sur les façons d'aborder les différentes étapes du cursus du mobilier: le prélèvement, le conditionnement, le lavage, le marquage, le stockage...; parfois aussi sur l'emballage, la manipulation et la confection de supports.

Pour répondre au fatalisme et aux arguments d'ordre économique, la formation en conservation préventive, pour être efficace, se doit d'insister sur les moyens d'éviter les dégradations par des actions appropriées, en en montrant le caractère peu gênant/ réaliste/bon marché/adapté aux moyens disponibles sur un chantier archéologique, voire sur le gain de temps dans les phases d'étude qui compense celui «perdu» sur le terrain. On tentera également de mettre en évidence que ces actions ont des avantages sur le plan de l'étude des mobiliers, car elles permettent généralement une meilleure préservation des informations archéologiques. Quand ce n'est pas le cas, par exemple pour la consolidation de certains matériaux, on tentera d'explorer les modes de conciliation possibles. Enfin, dans la plupart des formations, on élargit le propos «conservation préventive " à la nécessaire collaboration entre conservateurs-restaurateurs et archéologues, dans la mesure où, quelle que soit la qualité de la formation, certaines actions seront toujours du ressort du spécialiste, en insistant sur le dialogue et la recherche des meilleurs compromis pour la conservation et l'étude. 


\section{Les moyens de la formation}

La sensibilisation et la formation à la conservation préventive en archéologie peuvent prendre différentes formes :

- en premier lieu, et sous une forme indirecte, l'information, au moyen de brochures, dépliants, posters et autres documents, plus ou moins étendus ;

- sous une forme directe, l'exposé illustré de photographies ou bien, préférentiellement, d'objets réels ;

- la démonstration suivie de la pratique.

Les contenus concernant l'altération des matériaux se prêtent mal à une information au moyen d'ouvrages, textes ou brochures, sauf à être luxueusement illustrés, car l'essentiel dans cette étape est l'observation et l'entraînement à la reconnaissance des échantillons (fig. $\mathbf{n}^{\circ} \mathbf{1}$ ). Même la présentation de photographies au cours d'exposés commentés souffre de l'absence des éléments originaux, et ne garantit pas la reconnaissance effective du mobilier sur le terrain.

Figure 1

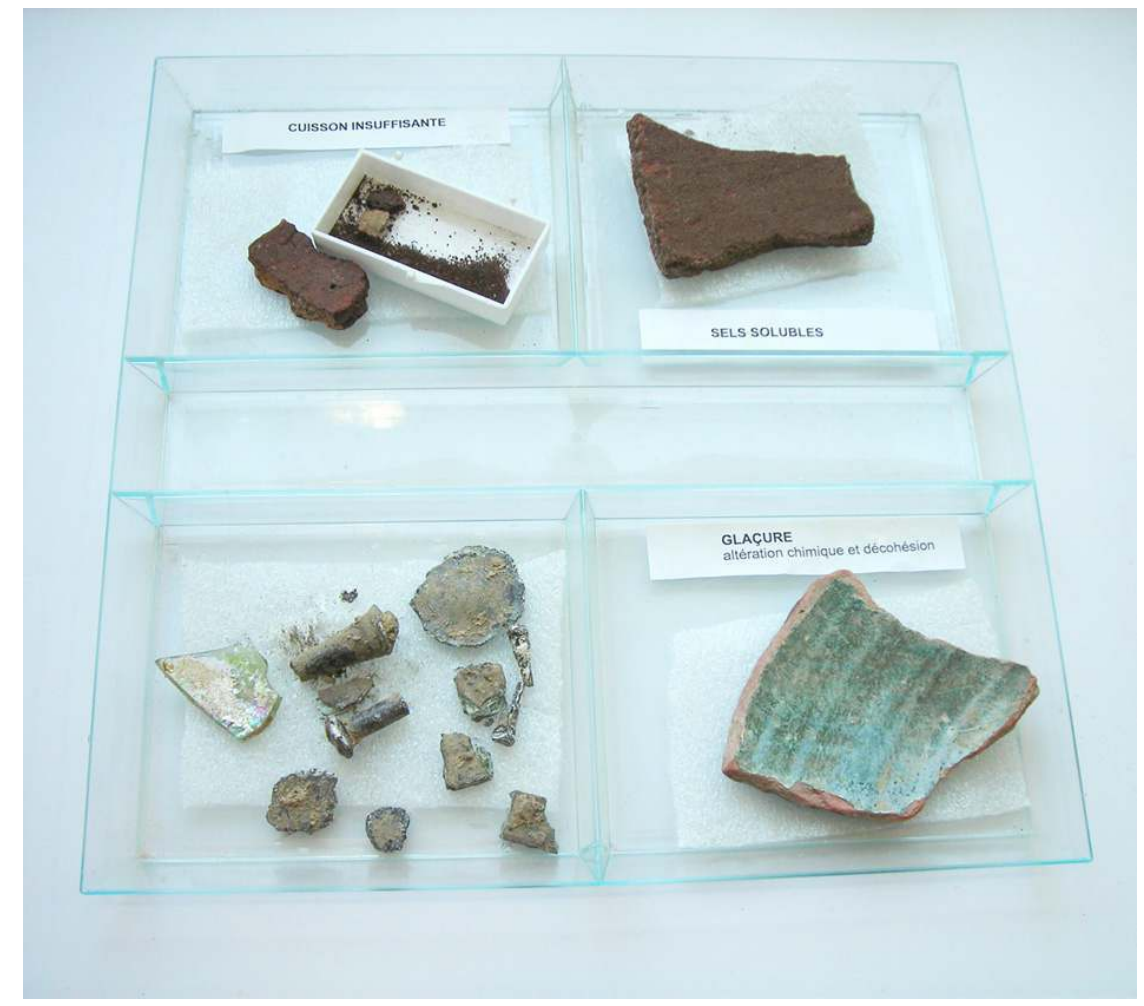

Plateau de démonstration des altérations de la céramique et du verre utilisé pour des formations au SADY. En haut à droite : tesson protohistorique, dont la moitié a été trempée dans l'eau (ce qui en est resté est dans la petite boîte...) ; en haut à gauche, un tesson présentant des efflorescences salines blanches ; en bas à droite : fragments de verre archéologique à différents stades d'altération ; en bas à gauche : altérations de la glaçure sur une céramique médiévale.

Phot. Païn, Silvia. (C) Service archéologique départemental des Yvelines, 2009.

Pour ce qui est des méthodes de dégagement et de protection des vestiges in situ et au cours du traitement archéologique du mobilier, il existe une pléthore de documents, proposés seuls ou en complément d'un exposé. De fait, beaucoup d'initiatives ont eu lieu 
dans ce sens, dont quelques-unes ont été publiées ${ }^{12}$, qui précisent les normes de conservation à long terme, les protocoles de traitement de telle ou telle catégorie de mobilier et/ou les modalités d'intervention sur le terrain. lors de ces formations (et un des seuls identifiés comme tels par les archéologues), peut difficilement être effectué spécialement pour une formation: les circonstances qui le rendent nécessaire ne se retrouvent pas à la demande... On pourrait à la limite envisager d'enterrer exprès des objets pour les faire prélever plus tard: ceci soulève quelques problèmes déontologiques si on travaille avec des objets patrimoniaux, des problèmes de représentativité si on travaille avec des objets modernes et des problèmes d'organisation dans tous les cas, raison pour laquelle on n'y a, à notre connaissance, presque jamais recours. L'apprentissage par l'exemple, au cours d'une réelle intervention, est plus viable et peut être mis en œuvre lorsque l'occasion se présente et que les conditions sont favorables (les délais sont confortables, le vestige se prête à un travail collectif,...). Toutefois, le stress induit par ce genre d'opération, même chez des professionnels expérimentés, n'est pas forcément propice à la pédagogie et, en tout état de cause, on peut difficilement former plus d'une ou deux personnes au cours de chaque intervention.

La formation à l'emballage ou à la confection de supports se prête mieux à une approche pratique et collective (avec des objets non patrimoniaux, bien entendu!, mais bons substituts) : emballer des objets, jeter la caisse par terre violemment pour simuler un choc, ouvrir la caisse et constater le résultat des soins mis à l'emballage est une expérience que certains de nos collègues n'oublieront pas de sitôt !

\section{Efficacité et perspectives de la formation des archéologues à la conservation préventive}

Il faut bien l'avouer: il est assez difficile de mesurer de façon précise l'efficacité des actions de formation en conservation préventive. Lorsque la formation s'est déroulée en interne, dans le service auquel on appartient, on peut, certes, vérifier concrètement si les conseils donnés sont ou non appliqués. Cependant, il faut bien se rendre compte que, dans ces cas, la plupart du temps, l'organisation du service est faite en prenant comme présupposé que les normes de l'institution comprennent les principes de conservation préventive, que les emballages et conditionnements conseillés sont fournis par la structure, ..., bref, toute l'infrastructure est en harmonie avec ces principes. Il est donc assez logique que l'intégration des normes de conservation préventive soit plus facile.

D'autre part, de par la présence même de personnel spécialisé en son sein, certaines actions sont naturellement déléguées à celui-ci et ne sont plus mises en œuvre par les archéologues eux-mêmes, mais par les gestionnaires des collections et/ou les conservateurs-restaurateurs présents. Aussi, les membres de l'équipe ayant été formés baignent dans une organisation où la démarche de conservation est intégrée à plusieurs niveaux, ce qui ne permet pas réellement d'apprécier quel serait leur comportement s'il leur appartenait de la mettre en œuvre de façon autonome. 
38 La plupart des expériences de formation en interne dont l'auteur a pu avoir connaissance - elles sont très rarement publiées - semblent à cet égard plutôt positives, mais des résistances ont certainement été rencontrées : comme le disent diplomatiquement Élise Alloin et Agathe Mulot ${ }^{13}$, «les agents ne sont pas tous réceptifs aux méthodes mises en place et une approche pédagogique personnalisée est nécessaire »...

Pour ce qui est des stagiaires formés en dehors de la structure de rattachement des formateurs, nous avons deux sources d'information : les réactions à l'issue du stage et les éventuels échos et consultations sur le court/moyen terme. La plupart du temps, ces réactions sont également très positives et reflètent souvent le sentiment d'avoir découvert un domaine largement méconnu. Le ressenti des stagiaires à l'issue de la formation, naturellement, ne nous dit rien sur l'acquisition effective des contenus transmis. En revanche, les questions que des anciens stagiaires peuvent poser donnent une idée, par leur pertinence, du niveau de réflexion auquel, grâce aux contenus et méthodes transmis, on a pu les amener. Cependant, comme, précisément, les formations ont pour objectif de répondre aux questions les plus généralement posées, l'absence de demande de conseils ultérieure n'implique pas l'absence de questionnements... Pour toutes ces raisons, l'évaluation des actions de formation reste une question complexe et délicate à résoudre. En revanche, l'écoute des questions lors des formations ou des remarques ultérieures peut donner quelques pistes pour mieux cibler les formations ou les améliorer.

\section{À qui s'adresse la formation?}

Concrètement, les bénéficiaires potentiels de formations sur la conservation préventive en archéologie appartiennent aux catégories suivantes :

- archéologues de terrain (quel que soit leur niveau hiérarchique) ;

- chefs de service ou d'opération (en tant que tels);

- archéologues spécialistes de l'étude des mobiliers ;

- gestionnaires de mobilier/responsables de dépôt.

La plupart des formations effectuées, animées soit par l'auteur de ces lignes, soit par des collègues, s'adressait aux archéologues de terrain. Celles issues du cahier des charges AFAN-ACREA étaient au départ destinées aux responsables d'opération au premier chef, puis aux responsables de secteur et gestionnaires de mobilier. Celle que nous avons animée au CNFPT, intitulée «La conservation préventive et les opérations de fouilles archéologiques » était proposée aux archéologues de collectivité territoriale en général : nous avons constaté une proportion importante de chefs de service et, curieusement, peu de gestionnaires de mobilier parmi les participants.

Malgré les difficultés qu'il peut y avoir à organiser des formations différenciées pour des publics spécifiques dont l'effectif peut se révéler dramatiquement faible, il nous semble, à la réflexion, que ces publics n'ont pas nécessairement besoin des mêmes contenus (même s'il y a des domaines de recouvrement) et, surtout, que l'on ne peut les transmettre de la même façon lorsque le but est la sensibilisation ou l'information que lorsqu'il s'agit d'acquérir une pratique professionnelle. Par exemple, pour l'archéologue de terrain, les problématiques de conservation à long terme (organisation du dépôt archéologique,...) relèvent de l'information, alors que les prélèvements sur le terrain relèvent de l'action. 
47 D'autre part, tous les contenus ne sont pas également faciles à transmettre et certains touchent inégalement les archéologues en fonction de leur spécialité. Il est souvent utile, au cours de ces formations, de faire appel à l'expérience des participants (cas heureux ou malheureux, difficultés rencontrées,...), de façon à ancrer l'exposé dans le quotidien du chantier archéologique (ou du post-fouille) tel qu'il se déroule concrètement.

L'objectif en matière de conservation préventive, idéalement, serait de toucher tous les personnels concernés. Dans la pratique, les formations ne touchent qu'une fraction assez faible de leur public, pour plusieurs raisons :

49 - le temps consacré à la formation n'est pas illimité, et d'autres thématiques apparaissent comme prioritaires ;

50 - l'offre de formation n'est pas très étendue et reste confidentielle;

51 - l'effectif étant faible en nombre et très distribué géographiquement, il est difficile d'organiser des formations peu onéreuses en termes de déplacements ;

52 - les problèmes de disponibilité aux dates prévues empêchent parfois des personnes intéressées de bénéficier des formations proposées ;

53 - on privilégie le volontariat pour l'inscription à ces formations (ce qui est logique, car, d'expérience, il est très difficile de former des personnes qui ne souhaitent pas l'être).

On pourrait également réfléchir à une formation «de proche en proche», c'est-à-dire, assurer une formation approfondie pour les gestionnaires du mobilier ou un archéologue de terrain référent pour les problématiques de conservation, chacun étant chargé de transmettre l'information aux autres membres de l'équipe. Il nous est arrivé, après un stage, d'être sollicitée sur la fourniture des présentations numériques illustrées dont nous nous étions servie, pour que les participants puissent à leur tour former leurs collègues. Cette modalité de formation en cascade à l'avantage de permettre de toucher plus de personnes sans les sortir de leur contexte de travail, ainsi que de responsabiliser et motiver les "passeurs». Son seul inconvénient est le risque de mauvaise interprétation des contenus reçus et, du coup, de transmission partielle ou erronée. Elle reste une piste très intéressante pour les raisons invoquées, mais aussi, dans la mesure où le gestionnaire du mobilier est aussi un archéologue, pour éviter que les personnels à former ne ressentent négativement la remise en cause de leurs pratiques professionnelles du fait qu'elle émane d'un professionnel d'un autre domaine.

\section{Où s'arrête la conservation préventive ?}

Lors de ces formations, celui qui anime le stage est amené à définir, dans le champ de la conservation, ce qui est de la responsabilité des archéologues et/ou des conservateursrestaurateurs; ce qui relève de la conservation préventive et/ou de la conservation curative. Il parait essentiel de montrer que chaque domaine implique un ensemble de compétences différent: les restaurateurs ne se veulent pas archéologues; les archéologues ne sont pas des restaurateurs. Il ne s'agit pas ici d'un problème de "pré carré ", mais de capacité à analyser une situation et à y apporter des solutions adaptées : dans certains cas, on peut envisager, sans avoir besoin de compétences particulières, des actions de conservation qui sont dans l'immense majorité des cas bénéfiques et rarement contre-indiquées; dans d'autres, seul un constat d'état approfondi et une bonne connaissance des problématiques d'altération et de leurs cinétiques peuvent mener à un diagnostic et à une proposition d'intervention qui, dans d'autres circonstances et sur 
d'autres objets, pourrait être néfaste... En effet, le problème n'est pas de savoir appliquer une recette, mais de savoir dans quelles situations cette recette est indiquée et dans quelles autres elle donne des résultats catastrophiques - ou ne peut être mise en œuvre. Malheureusement, dans les contextes réels dans lesquels se déroulent les activités archéologiques sur le terrain, deux problèmes se posent : le premier est que l'archéologue est celui qui découvre le vestige fragile, qui doit assumer la responsabilité de détecter les situations où il n'est pas compétent pour agir, et qui doit dire quand il a besoin de l'aide d'un spécialiste. Le deuxième est que, notamment s'il n'y a pas de conservateurrestaurateur dans l'équipe, face à un objet fragile qui doit être prélevé en motte ou consolidé, le choix n'est pas toujours entre faire soi-même ou appeler un conservateurrestaurateur, mais entre faire soi-même ou laisser détruire le vestige...

Pour ce qui est du premier problème, il pourrait sembler utile, lors des actions de formation, de tenter de définir les "indicateurs de complexité » qui permettraient à l'archéologue de terrain d'alerter sur la nécessité de s'adjoindre des compétences spécifiques en conservation, ce qui n'est pas nécessairement si facile ou si évident à faire de façon théorique. Il paraît essentiel, dans ce but, d'apporter des éléments d'analyse assez fournis, avec des mises en situation et des exemples concrets, pour permettre aux personnes formées de se rendre compte (en fonction également de leur propre expérience) s'ils sont capables de prendre en charge la problématique de conservation posée ou s'ils doivent " passer la main ».

Pour ce qui est des situations d'urgence où le concours d'un professionnel n'est pas possible dans des délais raisonnables, l'on pourra difficilement assigner aux archéologues les actions de conservation préventive (et seulement celles-ci) et aux conservateursrestaurateurs les actions de conservation curative et celles où l'interprétation fine d'un constat d'état est essentielle... Le formateur (surtout s'il est lui-même conservateurrestaurateur) se trouve donc dans une situation inconfortable, soit en refusant de donner à ceux qui sont sur le terrain les moyens de sauver des objets, s'il s'en tient strictement à une répartition des tâches conforme aux compétences, soit en donnant des conseils qui, dans l'hypothèse d'une appréciation erronée des risques et des situations, pourraient se révéler préjudiciables (mais aussi donner à ceux qui les suivent l'illusion d'une compétence qu'ils ne possèdent pas réellement).

$\mathrm{Si}$, lorsque l'on forme des collègues de travail, on peut observer la façon dont nos conseils sont appliqués et proposer des échanges ou formations complémentaires si l'on constate des difficultés, lorsque l'on dispense une formation à des archéologues d'autres institutions le message est délivré une fois pour toutes, et on n'a plus de prise sur la façon dont il a été compris ni sur la pratique concrète qui en découle. Il y a donc là un choix philosophique qui n'est pas toujours facile à trancher et pour lequel les réponses sont assez diversifiées. On remarquera, sur deux documents aux objectifs comparables ${ }^{14}$, tous deux rédigés par des conservateurs-restaurateurs à l'intention des archéologues pour une utilisation autonome (hors situation de formation), des partis pris différents pour ce qui est de la consolidation sur le terrain: la plaquette de l'INRAP donne des conseils pratiques, tout en disant que celle-ci doit rester «exceptionnelle»; le poster « De la fouille au dépôt » préfère renvoyer à un conservateur-restaurateur et n'apporte pas les moyens de la mettre en œuvre.

60 À titre personnel, nous avons toujours considéré que le risque de voir des connaissances mal employées, ou bien de croire tout savoir, était secondaire face au risque de laisser 
détruire un objet par manque de connaissances permettant de le sauver, alors même qu'on en avait la volonté : celui qui est en situation d'agir doit, à notre avis, savoir le faire et avoir les outils techniques et cognitifs pour le faire à bon escient et efficacement. Ainsi, nous avons donné place, dans les formations dispensées, à des conseils qui dépassent parfois le domaine de la conservation préventive pour les situations d'urgence sur le terrain, telle, notamment, la consolidation des vestiges qui le nécessitent (fig. $\left.\mathbf{n}^{\circ} \mathbf{2}\right)$.

Figure 2

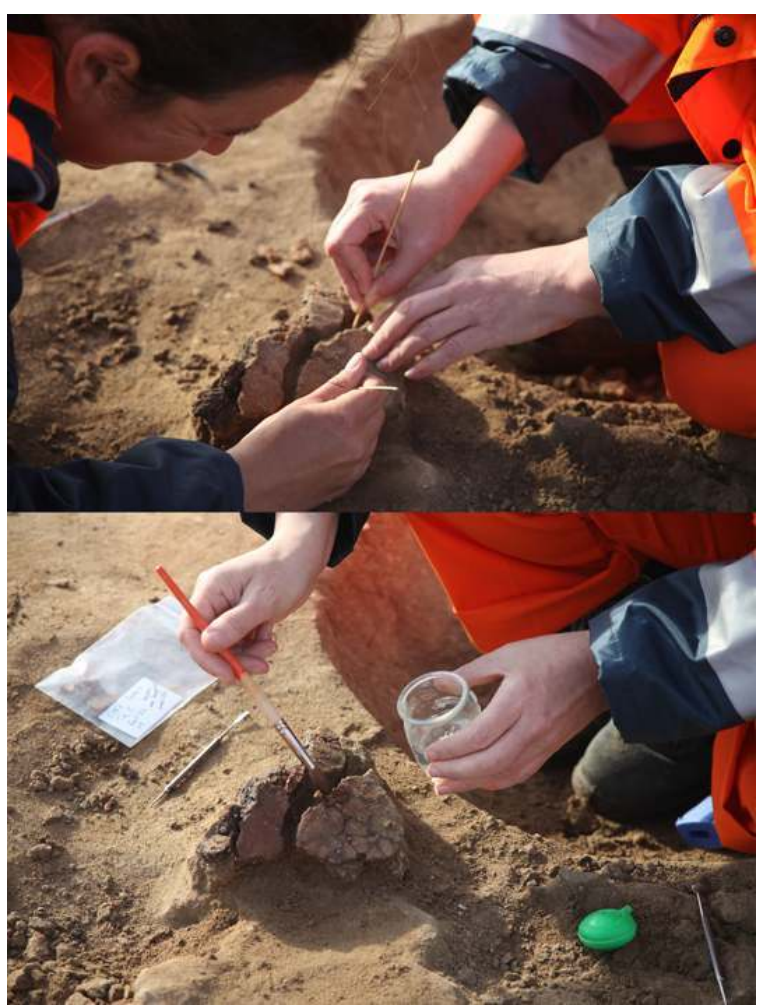

Dégagement et consolidation d'une céramique néolithique très fissurée. Le dégagement est effectué par l'archéologue (à gauche) et la restauratrice. C'est cette dernière qui effectue la consolidation, mais l'archéologue observe le geste, de façon à être à même de le reproduire en cas d'absolue nécessité.

Phot. Laforest, Pascal. @ Service archéologique départemental des Yvelines, 2009.

61 Les problèmes de conservation se posent non seulement sur le terrain, mais aussi au cours du traitement archéologique des vestiges... Le lavage et le séchage sont, en particulier, des moments clés où se joue parfois la survie, sinon des objets, parfois d'un décor ou d'un traitement de surface, voire la possibilité d'un remontage. Il importe, là aussi, d'insister sur "où et quand s'arrêter ", sur les matériaux qui ne doivent pas être lavés, ou bien sur les précautions à prendre au cours du lavage. Ici encore, ce sont les archéologues qui ont le premier contact avec le mobilier archéologique, et ils doivent avoir les moyens de déterminer si les traitements habituels sont indiqués ou si l'objet doit être confié à un spécialiste. Tout l'enjeu d'une formation à la conservation est d'amener les archéologues à avoir « les bons réflexes » en ce domaine, alors même, comme on l'a dit précédemment, que la frontière est difficile à cerner dans l'abstrait.

62 Certains archéologues sont parfois demandeurs de conseils pour nettoyer les monnaies, voire les objets en fer... Faut-il aussi y faire droit? Certaines expériences de ce genre ont abouti à des résultats pour le moins mitigés (objets décapés détruits par la corrosion, 
nettoyages chimiques non rincés ayant causé des altérations irréversibles,...). Il nous semble que, pour ce qui est des situations où la survie de l'objet n'est pas en cause, rien ne justifie que des interventions dépassant le cadre strict de la conservation préventive soient prises en charge par des non-professionnels, et nous nous employons activement à décourager ces initiatives et à en montrer les dangers au cours des formations effectuées. À ceux qui diraient que les délais de rendu du rapport constituent aussi une « urgence ", nous répondrions qu'il s'agit là peut-être d'une urgence, certes,... pour l'archéologue, (luimême contraint par une réglementation), mais nullement pour l'objet, contrairement aux situations de prélèvement sur le terrain, où les vestiges fragiles sont objectivement en danger. Pour autant, ce sentiment d'urgence et le besoin ressenti par l'archéologue doivent être pris en compte, et le formateur doit tenter de répondre à ces questionnements en proposant une conciliation ou une alternative. Pour ce qui est des objets en fer, par exemple, dont les délais de traitement de stabilisation dépassent le plus souvent ceux du rendu du rapport, du moins pour des diagnostics ou des opérations courtes, le recours à la radiographie $\mathrm{X}$ peut être présenté comme une possibilité d'identifier un certain nombre d'objets avant nettoyage, et même d'en effectuer un dessin archéologique d'après le cliché.

63 Est à cultiver chez les archéologues, en revanche, l'intérêt pour la conservationrestauration au sens large et la compréhension de ses apports au travail archéologique, dans le but d'arriver à un partenariat intelligent entre les différents acteurs de la conservation en archéologie. Les réactions à l'issue des stages montrent que ce qui a le plus marqué les participants n'est pas nécessairement le contenu directement orienté vers la conservation préventive (qui est pourtant l'objectif de la formation) mais, très souvent, la découverte du domaine de la conservation-restauration, largement méconnu, sinon ignoré, auparavant.

\section{Conclusion : vers une démarche de conservation intégrée?}

La formation en conservation préventive est une rencontre entre l'offre des formateurs et la demande des archéologues. Cette dernière est souvent orientée vers la résolution de problèmes, et les réactions après stage montrent que les participants apprécient les conseils directement utilisables et souhaitent souvent augmenter le temps consacré aux formations pratiques sur des gestes concrets. Il est difficile, dans ce cadre, d'échapper au piège des «recettes » et d'orienter la formation sur ce que pourrait être une démarche globale de conservation, du terrain au stockage, voire de susciter une conscience patrimoniale. C'est pourtant une ambition à nos yeux légitime, car pouvant conduire à une autonomisation des acteurs. En effet, si la démarche de conservation est assumée par les archéologues, le spécialiste de la conservation-restauration n'est plus le distributeur de conseils ponctuels (ou, à l'occasion, le donneur de bons et de mauvais points), mais un partenaire consulté à bon escient. La conservation n'est plus un aspect extérieur, «en plus ", subi, mais une dimension de l'archéologie, pleinement assumée et intégrée aux pratiques (fig. $\mathbf{n}^{\circ} 3$ ). 


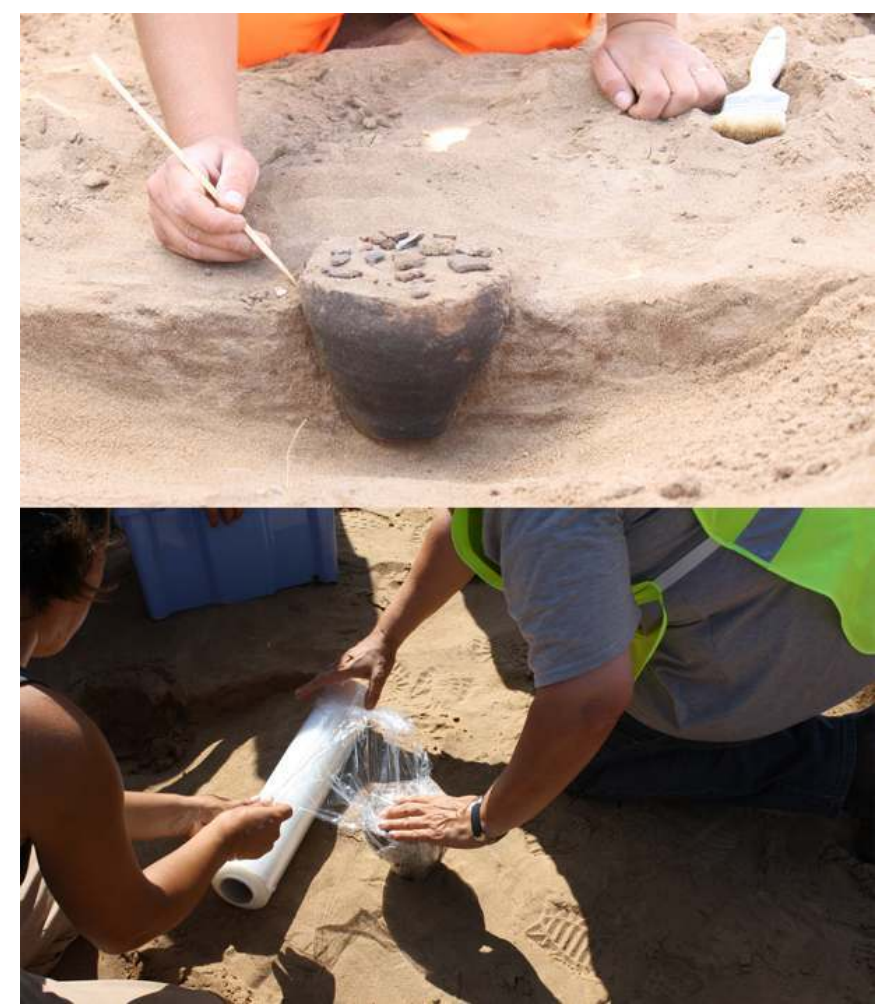

Une urne gauloise fissurée. En haut, l'urne en cours de fouille. En bas, le prélèvement par emballage à l'aide de film étirable est effectué par deux archéologues. Ces soins ont permis la conservation de l'objet pendant le transport et ont facilité son traitement en laboratoire.

Phot. Laforest, Pascal. @ Service archéologique départemental des Yvelines, 2009.

Comment, dans une action de formation, susciter cette démarche? Il s'agit de mettre en place une méthodologie globale, qui trouvera des applications particulières, mais qui doit avoir une cohérence intellectuelle et organisationnelle sur laquelle il faut insister lorsque l'on aborde chaque étape particulière. Pour ce faire, il faut décrire le cursus du mobilier comme un ensemble d'actions successives, ayant des caractéristiques spécifiques vis-à-vis des problématiques de conservation: certaines sont par nature des situations instables (l'exhumation, typiquement), d'autres sont théoriquement stables (le stockage de long terme), d'autres impliquent une modification volontaire et maitrisée (le lavage, le séchage). La démarche de conservation tiendra compte de la dimension temporelle de ces étapes (y compris des délais d'attente entre étapes), confrontée aux facteurs d'altération en présence, de façon à déterminer les risques réels, les prendre en charge par des actions adaptées et, selon les situations, dimensionner les moyens de prévention en fonction des délais prévus ou l'inverse.

Une telle démarche se décline ensuite en plusieurs interventions avec, pour chacune, une méthode basée tout d'abord, sur l'analyse de la situation (l'objet, le contexte), aboutissant à une estimation des risques, un projet d'intervention, l'intervention puis son évaluation, indispensable facteur d'amélioration.

67 Lorsqu'une telle démarche est donnée comme norme dans une institution, il est, bien entendu, plus facile de la faire vivre dans la durée et, en particulier, de l'évaluer sur le long terme. Essayer de la susciter au cours d'une formation en externe, nécessairement resserrée sur quelques jours et devant être mise en œuvre dans des contextes diversifiés, 
comme nous l'avons fait dans un stage du CNFPT, est moins facile, et le succès en est moins assuré. Cependant, il nous semble que cela mérite d'être tenté car, compte-tenu des enjeux, seule une démarche de conservation intégrée à l'activité archéologique et assumée par tous ses acteurs peut, à terme, garantir une réelle prise en charge de la préservation du mobilier archéologique.

\section{NOTES}

1. - DUFA $\ddot{Y}$, Bruno. «La conservation du mobilier et de la documentation archéologiques comme pivot d'un projet scientifique et culturel ». Dans Le dépôt archéologique. Conservation et gestion pour un projet scientifique et culturel. Actes des Assises nationales de la conservation archéologique, Bourges, 26, 27 et 28 novembre 1998. Éditions de la ville de Bourges, 2000, p. 37-42. Les citations sont extraites des pages 38 et 37 .

2. - MEYER, Nicole. «Gérer le matériel archéologique ». Dans BERDUCOU, Marie-Claude (dir.). La conservation en archéologie. Paris : Masson, 1990, p. 418.

3. - DUFAŸ, Bruno. «La conservation du mobilier et de la documentation archéologiques comme pivot d'un projet scientifique et culturel ». Dans Le dépôt archéologique. Conservation et gestion pour un projet scientifique et culturel. Actes des Assises nationales de la conservation archéologique, Bourges, 26, 27 et 28 novembre 1998. Éditions de la ville de Bourges, 2000, p. 40.

4. - DESACHY, Bruno. De la formalisation du traitement des données stratigraphiques en archéologie de terrain. Thèse de doctorat en archéologie, université de Paris 1 , soutenue le 12 décembre 2008, vol. 1, p.19. Téléchargeable sur: http://hal.archives-ouvertes.fr/docs/00/40/62/41/PDF/ Desachy_these_volume_1.pdf [date de consultation : 07/03/2012].

5. - SERDON, Valérie. Armes du diable. Arcs et arbalètes au Moyen Âge. Rennes : Presses universitaires de Rennes, 2005, p. 131.

6. - RODRIGUES, Nicole. «Gestion et conservation du dépôt de l'unité d'archéologie de SaintDenis ». Dans Le dépôt archéologique. Conservation et gestion pour un projet scientifique et culturel. Actes des Assises nationales de la conservation archéologique, Bourges, 26, 27 et 28 novembre 1998. Éditions de la ville de Bourges, 2000, p. 308.

7. - DIDELOT, Catherine. Aide-mémoire technique portant sur la mise en œuvre de la conservation à long terme des mobiliers archéologiques et de la documentation scientifique au sein d'un centre de conservation et d'étude (CCE). Saint-Denis: UTICA, 2010, http://www.archeologie.culture.gouv.fr/pdf/ cce_aide_memoire.pdf; Ministère de la Culture et de la Communication, direction de l'Architecture et du Patrimoine, direction de Musées de France - Agence ABCD. Du dépôt archéologique au centre de conservation et d'étude (CCE) : programmation du projet scientifique et culturel. Guide méthodologique. Paris : MCC, 21 octobre 2008, http://www.archeologie.culture.gouv.fr/pdf/ cce_guide.pdf, [date de consultation : 07/03/2012].

8. - DRIEUX, Monique, QUEIXALOS, Innocence, RELIER, Caroline. « Formation des archéologues à la conservation préventive: résultats des stages et études critiques». Dans L'actualité de la conservation-restauration en archéologie : produits et techniques/conservation préventive. (CRBC-Cahier Technique $n^{\circ}$ 3). Paris : ARAAFU, 1998, p. 48-52.

9. - Plaquette Bon sens, bon geste et poster Traitement du mobilier archéologique: conseils et recommandations. INRAP, 2009 ; voir aussi BIRON, Marina. « Des outils pour mieux accompagner les objets archéologiques ». Dans Actualité de la conservation-restauration en archéologie et recherches 
en cours. Bilans et retours sur expérience. (CRBC-Cahier technique $\mathrm{n}^{\circ} 18$ ). Paris : ARAAFU, 2009, p. 49-53.

10. - ALLOIN, Élise, et MULOT, Agathe. «Interactions du dépôt archéologique et du laboratoire de restauration dans la chaîne de traitement et d'étude du mobilier archéologique au Pôle d'archéologie interdépartemental rhénan». Dans Actualité de la conservation-restauration en archéologie et recherches en cours. Bilans et retours sur expérience. (CRBC-Cahier technique $\left.n^{\circ} 18\right)$. Paris : ARAAFU, 2009, p. 42-48.

11. - Par exemple, au service archéologique des Yvelines, les tableaux sur le premier traitement et le conditionnement, élaborés pour une formation interne datant de 1990 et remis à jour, sont repris dans les Normes pour le versement des collections et de la documentation de fouille. Traitement, conditionnement, inventaire. Montigny-le-Bretonneux : SADY, 2003, 29 p.; sur le chantier-école de Coupéré, on reprend des documents distribués lors d'un stage qui s'est déroulé en 1997 (voir article cité en note 4) dans Méthodes de fouille et mode d'enregistrement des données. Manuel pour le chantier-école de Coupéré. Saint-Bertrand-de-Comminges: Unité toulousaine d'archéologie et d'histoire, 2004, 85 p.

12. - Voir, pour les plus récentes, l'article cité en note 10 et BIRON, Marina. «Des outils pour mieux accompagner les objets archéologiques». Dans Actualité de la conservation-restauration en archéologie et recherches en cours. Bilans et retours sur expérience. (CRBC-Cahier technique $\left.n^{\circ} 18\right)$. Paris : ARAAFU, 2009, p. 49-53, ainsi que DEYBER, Dominique. « De la fouille au dépôt : guide pour la conservation préventive du mobilier archéologique ». Dans op. cit., p. 54-62.

13. - Voir la conclusion de l'article dans ALLOIN, Élise, MULOT, Agathe. «Interactions du dépôt archéologique et du laboratoire de restauration dans la chaîne de traitement et d'étude du mobilier archéologique au Pôle d'archéologie interdépartemental rhénan ». Dans Actualité de la conservation-restauration en archéologie et recherches en cours. Bilans et retours sur expérience. (CRBCCahier technique $\mathrm{n}^{\circ} 18$ ). Paris: ARAAFU, 2009, p. 48. Pour des publications de l'ARAAFU (Association des restaurateurs d'art et d'archéologie de formation universitaire), voir le site : www.araafu.com.

14. - La plaquette de l'INRAP est citée en note 9 et sera bientôt sur le site de l'INRAP ( www.inrap.fr). Le projet de poster rédigé par D. Deyber, D. Guillemard, A. Della Monica, Caroline Relier et S. Païn, inédit en tant que tel, est présenté dans l'article de Dominique Deyber cité en note 12.

\section{RÉSUMÉS}

L'enjeu de la formation des archéologues à la conservation préventive est, en premier lieu, de sensibiliser les professionnels aux risques lors de l'exhumation du mobilier, de façon à susciter les actions de conservation pertinentes, mais aussi d'alerter sur les dangers inhérents à l'étude scientifique, souvent sous-estimés. On tentera de s'interroger sur l'efficacité des différentes actions de formation, avec pour horizon la mise en place d'une démarche globale de conservation cohérente, depuis le terrain jusqu'au stockage définitif.

The issue of training archaeologists in preventive conservation is, first, to raise professionals to risks in the exhumation of the objects, to arouse relevant conservation actions, but also alert them to the dangers inherent to the scientific study, often underestimated. We will discuss the 
effectiveness of the various actions of training, aimed to the establishment of a comprehensive process of coherent conservation, from the field to the final storage.

INDEX

Mots-clés : conservation préventive, archéologie, formation

\section{AUTEUR}

\section{SILVIA PAIIN}

Conservateur-restaurateur d'objets archéologiques au Service archéologique départemental des Yvelines depuis 1989, intervenante occasionnelle pour des formations en conservationrestauration et en conservation préventive auprès du l'INP, l'École du Louvre, l'université de Paris 1 et le CNFPT spain@yvelines.fr 